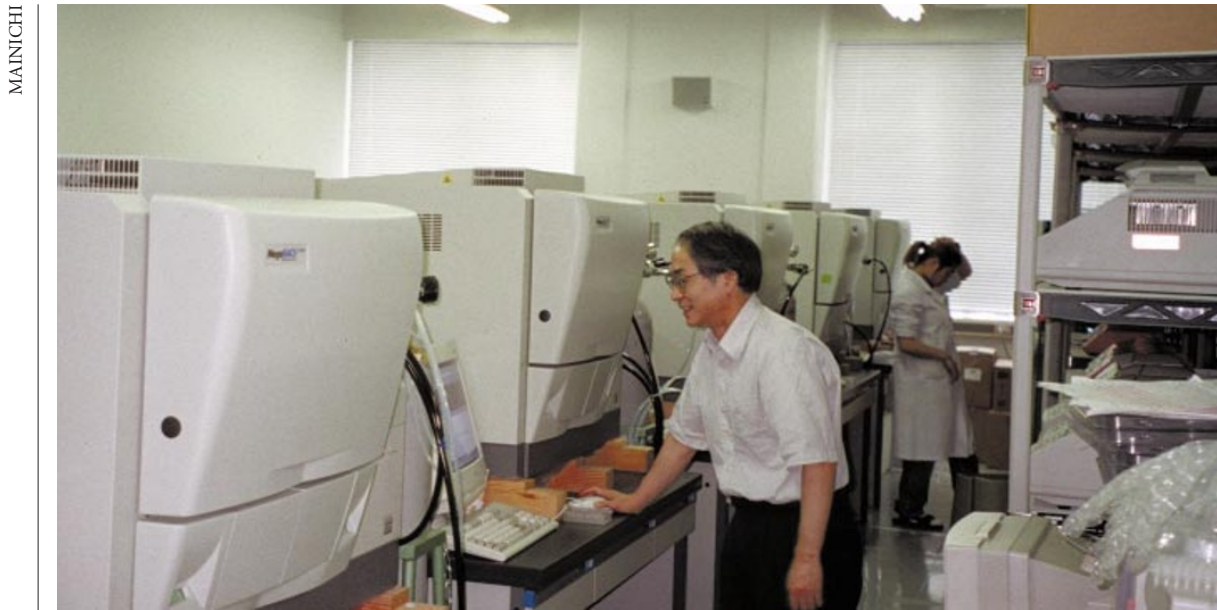

Sequencing solutions: but regulatory guidelines proposed so far have not been coordinated.

\title{
Japan seeks to unify ethics rules on genomics research
}

Robert Triendl, Tokyo

The Japanese government announced last week that is has created an expert advisory panel in a bid to coordinate the drafting of ethics guidelines for human genome research. The goal is to bring some order to a growing number of regulatory proposals issued by government agencies by establishing a common ethical ground for all such research in Japan.

According to government officials, the panel is expected to draft model guidelines "as soon as possible", and no later than December. Although these guidelines would not be binding, individual agencies would be expected to use them as a reference point.

The panel has been set up by the four agencies that fund most of Japan's human genome research: the Science and Technology Agency (STA), the Ministry of International Trade and Industry, the Ministry of Health and Welfare (MHW) and the Education Ministry (Monbusho). Most panel members come from the agencies' advisory committees.

The country's Millennium Project - a five-year plan targeting areas of science and technology with high economic potential has boosted funding for biomedical research, especially genomics. Following this, committees at various government agencies have drafted proposals for regulating the use of human material in research and protecting genetic information.

Advisory committees under the STA and MHW released guidelines for human genome research earlier this year. But so far regulation has been limited in scope and often applies only to research funded by a single agency or to activities within the Millennium Project.
An informal 'genome constitution' approved in late June by a subcommittee of the Council for Science and Technology, an advisory body close to the STA, has so far attracted little support from other agencies.

Critics in Tokyo argue that having the genomics guidelines drafted by an informal expert body without any legal status reflects fundamental problems in the Japanese approach to regulating biomedicine and biotechnology.

Flaws in the country's regulatory approach were revealed earlier this year when its parliament rejected a bill drafted by the STA to prohibit human cloning. Critics argued that the content of the proposed law was inadequate and its wording scientifically contentious.

According to Shohei Yonemoto, a policy analyst at the Mitsubishi Kasei Institute of Life Sciences in Machida near Tokyo, the committees drafting research guidelines in Japan typically lack the "capability, autonomy and legitimacy" needed in a field of regulation that is exposed to public scrutiny.

Japanese industry has kept a low profile. As the international standardization of approval procedures for clinical trials enables Japanese pharmaceutical companies to use data from trials in Europe or the United States in applying for drug approval in Japan, companies such as Yamanouchi have decided to carry out clinical trials in areas such as pharmacogenomics abroad.

Various industry organizations, including the Japan Biotechnology Association and the Japanese Pharmaceutical Manufacturers Association, have issued broad statements on the ethics of human genome research. But co Aิ areas with an unclear regulatory regime.

\section{The sky's the limit as radio telescope array is approved}

Steve Nadis, Boston

Small might be beautiful, but to astronomers bigger is often better. This is certainly true in radio astronomy, where international teams have now approved two giant arrays - the Atacama Large Millimeter Array (ALMA) and the Square Kilometer Array (SKA).

ALMA, scheduled to be built by the end of this decade on a high plateau in Chile's Atacama Desert, will have 64 12-metre antennas tuned to millimetre wavelengths, making it the world's largest ground-based telescope. But this will be dwarfed by SKA, a centimetre-wave radio array with a collecting area of a million square metres.

SKA, long a dream of radio astronomers, came a step closer to reality last week. At a meeting of the International Astronomical Union in Manchester, England, representatives from Australia, Canada, China, India, the Netherlands and the United States signed an agreement to bring SKA online by the middle of the next decade. The plan calls for choosing a design by 2005 and starting construction in 2010 .

Why the continual push for bigger telescopes? "This project can by justified by the rule of thumb that says you need something ten times bigger to make major discoveries in astronomy," says Barry Turner of the National Radio Astronomy Observatory in Charlottesville, Virginia.

"Three times bigger can get you better data, but ten times bigger might lead to important new findings."

Many challenges, including fundraising, lie ahead. "There is no pile of

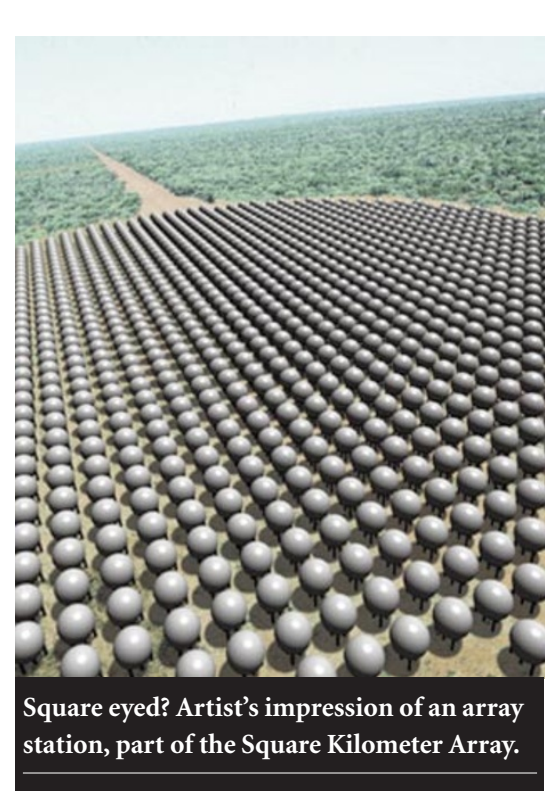


- money sitting on the table," says University of Manchester astronomer Peter Wilkinson, an early champion of SKA. "What we have is a resolve to pool our resources and a consensus that [after ALMA] this is the next big project in radio astronomy."

The ultimate location of the array is uncertain, with sites in Australia, Chile and China being considered. The success of the project will depend on whether researchers can find ways to reduce the cost by a factor of ten or more compared with conventional approaches, bringing the total to roughly a billion dollars.

There are several potential designs. Backers of the Allen Telescope Array, a one-hectare array named after the Microsoft billionaire Paul Allen who is giving it substantial funding (see Nature 406, 551; 2000), hope it will be a model for SKA. This telescope, which will link between 500 and 1,000 commercial radio dishes, is being developed by the SETI Institute and the University of California, Berkeley, for operation in 2005.

Australian researchers are investigating the use of 'refracting' telescopes called Luneburg lenses that would be deployed in 100 stations, each consisting of 400 lenses. The Canadian proposal is an antenna with a diameter of 200 metres that would reflect radio waves to a receiver suspended from a balloon 500 metres above the surface.

Chinese astronomers are contemplating a network of $\mathbf{3 0}$ radio antennas similar to the Arecibo telescope in Puerto Rico, a dish with a 300-metre diameter that sits in a natural depression. Researchers in the Netherlands favour a so-called phasedarray approach, consisting of millions of small antennas embedded in flat panels.

Given the technical and logistical uncertainties, Turner, for one, is sceptical of the timetable set for SKA at the Manchester meeting. "For every step of the way, there are so many choices the size of the dish, the target frequency range - all of which are subjects of sharp debate. They could get it all sorted out by 2005 , but I wouldn't count on it.”

But many in the field are confident that SKA will be worth the wait, even if it does not stick precisely to the schedule. For example, scientists are keen to spot irregularities in the distribution of the primordial hydrogen that gave rise to early galaxies.

And the telescope will also aid the search for extraterrestrial intelligence, enabling astronomers to examine more than a million Sun-like stars while listening for 'leakage' — random TV and radio signals - as well as messages sent intentionally.

\section{NASA pins hopes on bigger, costlier mission to Mars}

William Triplett, Washington

Is the era of 'faster, better, cheaper' space missions - NASA's mantra for the past decade - over? Last week the US space agency announced a new mission to Mars that will cost almost $\$ 600$ million, roughly twice the combined cost of its previous two missions to the planet.

The announcement has led to speculation that there has been a fundamental shift in the agency's thinking. It comes in the wake of an independent report, commissioned by NASA following recent failed missions, that questioned the 'faster, better, cheaper' policy.

Ed Weiler, NASA's associate administrator for space science, the division in charge of the new mission, says that there has been a shift in attitudes, but not a big one. "It's certainly an adjustment," he says. "But it's not a step back to the old days, when you did [a project such as] Viking and then waited ten years until you did anything else."

Like the pair of Viking landers that NASA successfully sent to Mars in the 1970s, the new mission will dispatch a pair of rovers, each about the size of a golf cart, in separate launches in 2003. If one fails, there is still the other; if both succeed, the mission gathers more scientific information.

NASA is desperate to avoid a repeat of its most recent mission to Mars, last year's loss of the Mars Polar Lander and Mars Climate Orbiter, which together cost $\$ 300$ million. The failures led to a review of NASA mission planning that concluded, as Weiler puts it, "that clearly 'faster, better, cheaper' had been pushed to its limits".

The successful Mars Pathfinder mission,

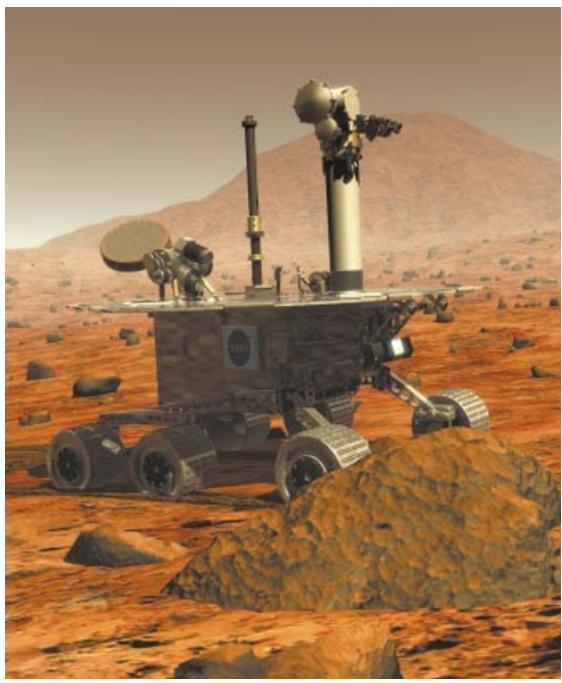

One of a pair: NASA will send two rovers to Mars in 2003 to search for signs of water.

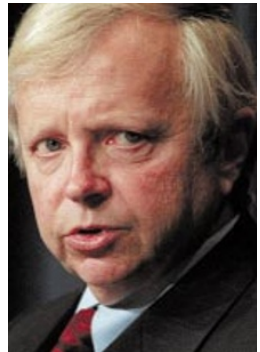

Weiler: acknowledges a shift in attitudes. which sent a single rover to the planet's surface in 1997, cost about $\$ 300$ million. "We then took the bolder step of doing two missions for the price of one," says Weiler. When these both failed, he adds, "it didn't take a rocket scientist to figure out that maybe we'd pushed too far on costs and raised risks."

But 'fast, better, cheaper' has not been completely ditched. Weiler points out that when adjusted for inflation today's cost of the Viking missions, with landers but no rovers, would be $\$ 2-3$ billion. "So is the new Mars mission still cheaper? You bet."

He also says that this is more than just a doubled repeat of Pathfinder. The mission's goal is to search for signs of water, either present or past, which scientists think might be on Mars. But Pathfinder, Weiler notes, covered only 100 metres of territory in three months. The new rovers "cover about a hundred metres a day".

Louis Friedman, executive director of the Planetary Society, agrees that the new mission signals an apparent shift in approach. But he is cautious about how significant the shift is. "The pendulum is definitely swinging back," Friedman says. "But where it will end up, I'm not sure."

He says that "all the space science news I've been hearing out of NASA is that a number of projects will be costing more now because of [the steps being taken] to ensure better reliability of space science missions. And it's pushing costs up to the point that there's a threat that other missions might have to be cancelled."

There have already been warnings about possible threats to the Pluto-Kuiper Express (see Nature 406, 554; 2000). If too many such missions are cancelled, Friedman says, NASA may indeed find itself back in the days of Viking, with missions few and far between - less expensive, perhaps, but the same in principle.

But John Logsdon, director of the Space Policy Institute at George Washington University in Washington DC, doubts whether there will be even a small return to NASA's old days. The new Mars mission, he says, is nothing more than "a mid-course correction based on hard experience". The biggest implication of last week's announcement, he argues, is that it demonstrates NASA's continued commitment to exploring Mars. 